\title{
CAPACITY IMPROVEMENT ANALYTICAL TOOLS AND BENCHMARK DEVELOPMENT FOR TERMINAL OPERATIONS
}

\author{
Seamus M. McGovern, U.S. DOT National Transportation Systems Center, Cambridge, Massachusetts
}

\begin{abstract}
With U.S. air traffic predicted to triple over the next fifteen years, new technologies and procedures are being considered to cope with this growth. As such, it may be of use to quickly and easily evaluate any new technologies or procedures against a set of benchmarks, including best case and worst case. In this paper, theoretical upper and lower capacity bounds are formulated along with a performance index equation to allow for the quantitative comparison and evaluation of different terminal area capacity-increasing enhancements. These benchmarks are then used with a conventional stochastic queueing model $(M / D / 1)$ and a selected capacity improvement in order to demonstrate how an example change impacts the terminal airspace capacity as compared to the theoretical worst case and best case. These mathematical tools may hold value in quantifying proposed technologies and procedures intended to improve efficiency and to absorb the predicted increase in air transportation, prior to any actual implementation.
\end{abstract}

\section{Introduction}

According the Joint Planning and Development Office, by 2025 U.S. air traffic is predicted to increase two to three times, with the traditional air traffic control system expected to be unable to manage this growth [1]. The Next Generation Air Transportation System (NextGen) proposes active networking technology that updates itself with realtime shared information and tailors itself to the individual needs of all U.S. aircraft, allowing for adaptability by enabling aircraft to immediately adjust to ever-changing factors such as: weather, traffic congestion, aircraft position via GPS, flight trajectory patterns, and security issues [1]. With an influx of new technologies, it may be of use to evaluate these against formalized benchmarks, to include upper and lower bounds. In this paper, theoretical upper and lower capacity bounds are developed along with a performance index formula to allow for the quantitative comparison and evaluation of different changes to terminal capacity-increasing enhancements.

This paper first provides a summary of both the problem and the literature to date, as well as a short introduction to the field of queueing theory. A queueing theory-based model is then proposed to describe the final approach phase at an airport along with formulations for calculating the theoretical benchmarks. Next, a set of equations to measure performance as compared to best-case and worst-case benchmarks is created. A case study is then used to demonstrate the application of the model and the proposed mathematical formulations in order to demonstrate the significance of the impact made to the terminal airspace capacity as given by comparison to the theoretical worst- and best-case bounds. Analysis conducted on the results using the model and bounds to quantitatively describe the impact of changes in various parameters.

The tools proposed here may hold value in preemptively quantifying proposed NextGen (or other) procedures or technologies that are intended to improve efficiency and mitigate the predicted increases in air transportation.

\section{Background}

Airport capacity is a critical contributor to the overall National Airspace System (NAS) capacity. It can be defined in various ways: it can be measured by the number of arriving aircraft, departing aircraft, total aircraft, passengers, specific aircraft types or sizes, etc. It may also be defined using some measure of time; that is, some measure under consideration taken over a specified unit of time (e.g., takeoffs per hour), time of day (usually, the peak times), or time of year (i.e., seasonal). Whatever measure is used, an airport's capacity depends on a variety of considerations. Ginsburg [2] lists five general areas affecting airport capacity: the size of the aircraft, approach and departure paths, safety, weather, and other. Aircraft size determines takeoff distances, braking distances, approach speeds, turning radii, ramp space, and ultimately runway

U.S. Government work not protected by U.S. Copyright. 
selection and usage. Approach and departure paths can limit the use of the multiple runways available at larger airports. In addition, the geographical location of an airport can further restrict the approach and departure paths, including those due to emissions (i.e., noise or pollution) considerations. Safety can also impact efficiency, especially in the terminal area since landing and take-off are traditionally considered to be the portions of operations having the greatest safety risk. Atmospheric conditions can have an impact both on terminal operations and on aircraft performance. These items can include icing, visibility, winds (including crosswind considerations), pressure, temperature, humidity, convective action, etc. Finally, general areas having an impact can include congestion, delays, accidents, unexpected acts (intentional damage to aircraft or communication, navigation, and surveillance systems; worker strikes; etc.), passenger emergencies, human error, unusual weather, hardware or software failures, etc. Any strategy for dealing with these can have an impact on the airport's capacity and associated delays. Ginsburg categorizes these strategies as: arrival/departure sequencing, multiple runways, runway configuration, buffers (blocks of time between arrivals and departures to prevent the violation of safety rules and regulations), and delay recovery.

Separations are required between aircraft to ensure safety. These separations may depend on the aircrafts' routes, weights, speeds, etc. Efficient sequencing of the terminal aircraft can reduce the total separations and increase throughput. Regardless of conditions, the controller is responsible for implementing the arrival or departure scheduling, as well as all communication and safety monitoring. As a result, this is recognized as a very intensive job, while the high workload acts to limit the number of aircraft that the controller can take account of when sequencing. With technologies and procedures being proposed to assist both pilots and controllers, while maintaining or increasing safety and increasing throughput, it is of interest to know how much of an improvement these new NAS components can provide before the costs of equipage and operator training are incurred by either the airlines or the Government.

With the goal of any service system being to gain as much efficiency in service time while limiting queue sizes (i.e., waiting lines), number of balkers (customers that cannot get into a system to due capacity constraints), and minimizing costs, aviation researchers have often used queuing theory to determine how to best utilize limited NAS assets. In this paper, queueing theory is used to model the approach portion of the terminal area, and then metrics are developed along with upper and lower bound formulations on these metrics to quantify performance improvements from any new procedure or technology. Finally, the queueing model and the metrics are combining and applied with case study data to demonstrate the use of these models and metrics.

\section{Literature Review}

Airport capacity is a richly studied area and includes a great deal of contemporary research. In the late 1970s, Newell [3] provided a well-written survey and critique of the literature on airport capacity. This study focused on how an airport's capacity depends numerous considerations including the sequencing of various types of operations (e.g., heavy or light aircraft, arriving or departing, etc.), runway geometry, flight conditions (i.e., visual flight rules or instrument flight rules), etc.

Beasley et al. [4] consider the problem of scheduling aircraft landings at airports as defined by deciding a landing time for each plane such that each plane lands within a predetermined time window while maintaining separation criteria. This was modeled using a mixed-integer zero-one formulation of the single runway case and extended to the multiple runway case. The problem is solved using linear-programming-based tree search. They then present a heuristic for the problem. Finally, computational results for both are presented for a variety of test problems involving up to 50 planes and four runways.

Janic [5] considers how large-scale disruptions of airline networks can cause deterioration of planned flight schedules, including delays, rerouting, and cancellation of flights. The study presents a model for the assessment of the economic consequences of these disruptions on a hub-and-spoke network as expressed by the cost of delays and cancellations. The model is based in queueing theory, modeling the airline hub airport as a server and the flights as customers in the queueing system and using the case study of a large European airline. 
Atkin et al. [6] makes use of the case of London Heathrow Airport. One of the busiest airports in the world, it only has one runway for use by departing aircraft at any time. With separations required between each pair of aircraft at take-off (depending on their routes, weights, and speeds) to ensure safety, efficient scheduling of the aircraft for take-off can reduce the total separations and increase throughput. Atkin et al. present models for evaluating a schedule and for determining the effects of the physical constraints imposed by the runway configuration and geometry. With this, a hybrid metaheuristic is proposed that can take into account more aircraft than a human controller can manage in order to recommend schedules for use by the controllers.

Another decision support tool is proposed by Venkatakrishnan et al. [7]. They consider the benefits to air traffic control as provided by decision support systems by studying air traffic delays for landing aircraft at Boston's Logan Airport. They develop an empirical model for landing-time intervals between aircraft as defined by the factors that most significantly affect them: runway configuration and the aircraft weight category. They also develop models of Boston's terminal airspace and apply sequencing algorithms meant to expedite the landing of incoming aircraft. Their results indicate that better sequencing can reduce delays by $30 \%$ in some instances, though at the expense of a possible increase in controller workload.

\section{Queueing Theory Background}

In the field of operations research, the two primary types of modeling techniques are prescriptive models (where the model prescribes a solution; e.g., linear programming) and descriptive models (where the model describes a situation to allow for analysis but does not provide a solution; e.g., queuing theory). A queueing (i.e., waiting line) theory model consists of a calling population and the queuing system (see Figure 1).

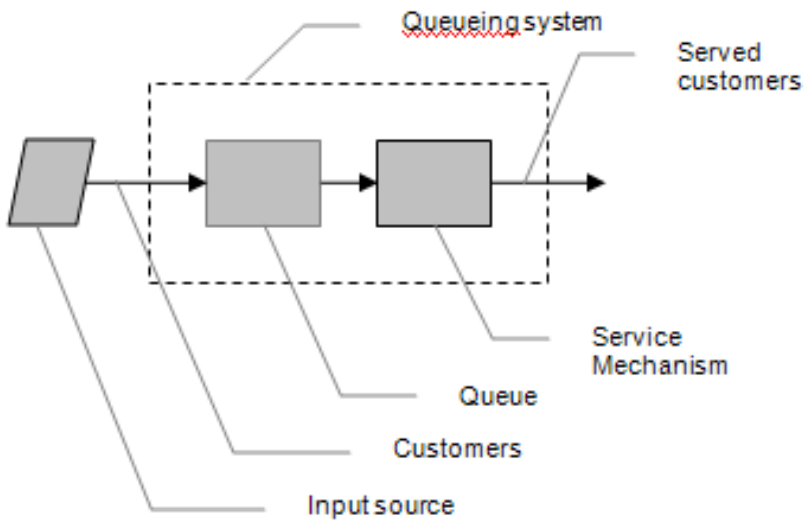

Figure 1. The Basic Queueing Process

The calling population characteristics include its size (finite or infinite) and generation pattern (or arrival rate; generally interarrival rates are assumed to be exponential, i.e., following a Poisson distribution). The queueing system is the portion of the model that deals with the queue and the service facility. The queue can be finite or infinite; the service discipline may be first in first out, last in first out, or random; the service facility is defined by the number of servers, their arrangement (series or parallel), and the service pattern (generally assumed to be exponential); exits from the system occur due to completion of service, balking, or reneging (leaving the system after joining). Model assumptions include (except for some specific variations): only one event can occur at a time, arrivals occur randomly and independently of other arrivals and according to a Poisson distribution, service times vary according to an exponential distribution, and that the system is past its transient period and has entered steady-state operation (i.e., it is independent of its initial state). Where arrivals follow an Poisson distribution, the probability of $x$ arrivals in a specific time period is given by

$$
P(x)=\frac{\lambda^{x} \cdot e^{-\lambda}}{x !} \text { for } x=0,1,2, \ldots
$$

where $\lambda$ is the mean number of arrivals per time period. Where service rates are exponential, the probability that the service time will be less than or equal to a time of length $t$ is given by 


$$
P\{\text { service time } \leq t\}=1-e^{-\mu t}
$$

where the service rate $\mu$ is the mean number of units that can be served per time period.

Several parameters describe the system. The state of the system $n$ is equivalent to the number of customers in the waiting line plus the service facility. $N$ is the maximum number of customers in the system. The probability of being in state $n$ (i.e., the probability of exactly $n$ customers in the system) is $P_{n}$. The length of the queueing system is given by $L$ (the expected number of customers in the queueing system), while the length of the queue itself is given by $L_{q}$. The expected time in the queueing system is represented by $W$ (the expected waiting time in line is represented by $W_{q}$ ). Little's formula gives $L=\lambda W$, $L_{q}=\lambda W_{q}$, and $W=W_{q}+1 / \mu$, where $1 / \mu$ is the average amount of time a customer spends in a service facility.

Kendall notation is used to depict a chosen model's characteristics. In the first portion of the sixtuple $a / b / c: d / e / f, a$ describes the arrival pattern (Markovian $M$, general $G$, degenerate $D$, Erlang $E_{k}$, etc.), $b$ describes the service pattern $\left(M, G, D\right.$, or $\left.E_{k}\right)$, and $c$ describes the number of servers in parallel. In the second portion, $d$ describes the service discipline (first come, first served FCFS, last come, last served LCLS, rotation, random SIRO, priority PRI, or any other GD), $e$ describes the storage capacity (the maximum number of customers allowed in the system; $N$ describes a limited capacity), and $f$ describes the calling population (size is finite or infinite). (Note that the default for the second portion is FCFS $/ \infty / \infty$; if this describes the model, it is often not included in its Kendall notation.) For example, $M / M / 1$ : FCFS $/ N / \infty$ (or $M / M / 1 / N$ ) is the Kendall notation for a single server, finite waiting line queueing system with Poisson arrivals and exponential service.

When queueing models are based on the birthand-death process (in the context of queueing theory the term birth refers to an input or arrival and the term death refers to a departure or a completed service), the system can be represented by a rate diagram. Set up as a Markov chain, a rate diagram is a conceptual model that describes the possible states of the model and the transitions from one state to another. The rate in = rate out principle can then be used to generate balance equations. Finally, since we have $N+2$ equations (given by the balance equations) and $N+1$ unknowns (given by the possible states of the rate diagram) the balance equations can be solved. These solutions, along with Little's formula, are then used to provide closed formulations of all parameters of interest. Additional background and further details are provided by Hillier and Lieberman [8].

\section{Queueing Theory-Based Model}

$M / M / 1$ models are often used to model airport capacity. A prototypical example by Hillier and Lieberman [8] considers the entire queueing system to include holding aircraft (the holding pattern is the queue), approaching aircraft as arriving customers which are cleared one at a time on the approach (i.e., only one aircraft is on approach, landing, then taxiing clear at any time - once that aircraft is clear of the runway, a holding aircraft can commence its approach), the runway as the server, a given arrival rate, and a service rate related to the time required for the aircraft to clear the runway. The goal of the example is to determine the average number of aircraft holding and average time in holding, with further study related to the effects of increasing the arrival rate and increasing the number of runways.

In this paper, a queueing model with a nonexponential distribution is used as an extension to the example by Hillier and Lieberman. An $M / G / 1$-based model (specifically an $M / D / 1$ model) is used to enable mimicking the separation provided by the air traffic controller. Once an aircraft has traveled a distance equivalent to the allowable separation interval (and well before reaching the runway, landing, and taxiing clear), the next aircraft is allowed out of the queue and into the service area (the approach). While both models consider that only landings are taking place on a given runway (i.e., no interfering takeoffs) and the queue is the holding pattern, the $M / G / 1$ model here differs from that in Hillier and Lieberman [8] and other studies by considering the service mechanism to be degenerate (i.e., deterministic) and allowing for multiple aircraft on approach since an aircraft is considered serviced and leaves the queueing system once it is a safe distance ahead of the following aircraft. 
As long as the mean $1 / \mu$ and the variance $\sigma^{2}$ of the service-time distribution are known, the $M / G / 1$ queueing formulations provide closed-form solutions to all parameters of interest. The $M / G / 1$ queueing formulation used for this terminal area model consists of the following familiar queuing theory equations: The probability of being in state zero (i.e., no aircraft in the system) $P_{0}$ is

$$
P_{0}=1-\rho, \quad \rho<1
$$

where the traffic intensity (also known as the utilization factor) $\rho$ is defined as

$$
\rho=\frac{\lambda}{s \cdot \mu}
$$

and $s$ is the number of servers (set to one here). Queue lengths and waiting times are given by

$$
\begin{gathered}
L=L_{q}+\rho \\
L_{q}=\frac{\lambda^{2} \cdot \sigma^{2}+\rho^{2}}{2 \cdot(1-\rho)} \\
W=W_{q}+\frac{1}{\mu} \\
W_{q}=\frac{L_{q}}{\lambda} .
\end{gathered}
$$

If it is of interest to consider differing arrival rates, the effective arrival rate $\lambda_{e}$ is then calculated using

$$
\lambda_{e}=\sum_{n=0}^{\infty} \lambda_{n} \cdot P_{n}
$$

Queueing theory provides a rigorous and accepted probabilistic methodology to measure the performance of waiting line systems and is often applied to transportation systems. Note that if constant arrivals and service were considered, a more appropriate model would be that of a flow shop thoroughly detailed by Pinedo [9]. It should also be noted that the queueing model proposed here is simply a tool selected to allow a comparison to be made in order to demonstrate this paper's capacity model, benchmarks, and performance metrics. Other queueing models can be used, as can deterministic models, other stochastic models, or simulation software (e.g., Arena, Simulink, etc.).

\section{Capacity Model}

Two obvious ways to measure terminal area performance include aircraft per hour and passengers per hour. Assumptions in the model proposed here include a flat earth (see Vincenty [10] for ellipsoid modeling); the approach indicated airspeed (IAS, or more typically, knots indicated airspeed KIAS) is always along the ground track (i.e., taken as parallel to the ground, not the glideslope or, more accurately, the aircraft's pitch attitude); the approach speed is the same as the groundspeed; constant approach speed (there is no deceleration on approach as is often practiced by controllers and pilots); and any additional runways that are in operation for approaches are parallel (therefore, multiple approaches are modeled by linearly increasing the analytical results). Most of these assumptions can be removed with some additional refinement (e.g., the airspeed assumptions) but are maintained here in the interest of making the mechanisms of the model the focus of this study. All measures are in nautical miles (NM) and hours. Also, the runway is not considered to be a bottleneck; i.e., the aircraft are considered to land, decelerate, and clear the runway at least as quickly as the service rate. While the model is general for any airport (using the previous assumptions), the parameter data used must be reflective of the airport being studied.

The service distribution mean $1 / \mu$ is obtained from the average approach speed and the average spacing by

$$
\mu=\frac{V_{e}}{\text { spacing }_{e}}
$$


where $V_{e}$ is the expected (i.e., average) approach speed and the expected aircraft spacing spacing $_{e}$ is given by

$$
\text { spacing }_{e}={\text { interval }- \text { length }_{e}}
$$

where interval is the controller-determined spacing between aircraft (actually, between aircraft centers of mass) and lengthe is the expected length of the aircraft on approach to a particular runway (length ${ }_{e}$ can be set equal to zero for low resolution systems or relatively large spacing, both of which are the case in the NAS at the time of this paper) [11]. For example, aircraft approaching at 120 knots and allowing for 3 $\mathrm{NM}$ spacing (using length $h_{e}=0$ ) results in 40 aircraft serviced per hour.

The concept of expectation can be found in most general statistics texts. This definition of average from the area of probability is commonly referred to as mathematical expectation $E$ and defined as

$$
E=x_{1} \cdot P_{1}+x_{2} \cdot P_{2}+\ldots+x_{n} \cdot P_{n}
$$

where the $x$ s represent some amount of interest (e.g., speed $V$ ) and the $P$ s represent the associated probability of obtaining those amounts.

If the parameter of interest is measured in passengers per hour, the service rate $\mu$ is given by

$$
\mu=\frac{V_{e} \cdot P A X_{e}}{\operatorname{spacing}_{e}}
$$

where $P A X_{e}$ is the expected number of passengers per aircraft. Using the example above, if each of those aircraft averaged 100 passengers, the landing rate would be 4,000 passengers per hour.

Since the service rates are modeled as being deterministic, the variance $\sigma^{2}$ of the service-time distribution is $\sigma^{2}=0$.

The arrival rate $\lambda$ describes the number of aircraft per hour. This value would be selected to be appropriate to the particular airport being studied and must adhere to the constraint that

$$
\lambda<\mu
$$

The airport's aircraft capacity $A C A P$ is the same as the airport's service rate $\mu$ and can be calculated in the same manner as equation (10):

$$
A C A P=\frac{V_{e}}{\text { spacing }_{e}} .
$$

This is similar for the airport's passenger capacity $P C A P$ which is calculated in the same way as equation (13):

$$
P C A P=\frac{V_{e} \cdot P A X_{e}}{\operatorname{spacing}_{e}}
$$

Using this model, it can be seen that terminal area capacity is primarily affected by aircraft spacing (less spacing allows more aircraft) and approach speed (faster approach allows more aircraft). (In the case of capacity being measured in terms of the number of passengers per hour, aircraft passenger capacity is also a factor.)

\section{Benchmark}

Theoretical upper and lower bound formulations provide the two extremes to be used as benchmarks for evaluation purposes. The benchmarks developed here are modeled deterministically and do not use queueing theory.

The minimum number of aircraft on approach in a terminal area each time period $A C A P_{\text {min }}$ is trivially zero and hence the theoretically lower bound and given by

$$
A C A P_{\min }=0 \text {. }
$$

This is analogous in the passenger capacity scenario with the minimum number of passengers serviced each time period $P C A P_{\text {min }}$ given by

$$
P C A P_{\min }=0 \text {. }
$$


The maximum number of aircraft would be obtained by aircraft touching nose to tail and doing so at a high approach groundspeed. While aircraft touching in flight would appear to be unrealistic, it is valuable in providing a theoretical upper bound. Also, previous capacity improvement proposals have included the concept of aircraft flying approaches in formation. Nose-to-tail spacing along the aircraft longitudinal axis (but not the lateral) is fairly commonplace in military formation flying using what's known as parade position, which provides aircraft separation using horizontal stagger and altitude step-down (step-up for helicopters) [12]. Also, limitations of formation operations in the NAS and potential solutions are detailed by Harrison [13]. From equations (11) and (15) the maximum number of aircraft on approach in a terminal area each time period $A C A P_{\max }$, the theoretical upper bound, is

$$
A C A P_{\max }=\max \left\{\frac{V_{x}}{\text { length }_{x}}\right\} \forall x
$$

with

$$
x: x \in\{\text { all aircraft }\} \text {. }
$$

$A C A P_{\max }$ is determined by the aircraft having the highest speed-to-size ratio on a given approach at a given airport.

This is analogous in the passenger capacity scenario, with the maximum number of passengers serviced each time period $P C A P_{\max }$ given by

$$
P C A P_{\max }=\max \left\{\frac{V_{x} \cdot P A X_{x}}{\text { length }_{x}}\right\} \forall x
$$

again, with

$$
x: x \in\{\text { all aircraft }\} \text {. }
$$

This value is determined by the aircraft having the highest speed-and-passenger-count product to size ratio on a given approach at a given airport.

While it would seem that equations (19) and (20) should more accurately incorporate the floor function (the floor function of $x$ assigns the largest integer $\leq x$, e.g., $\lfloor 1.3\rfloor=1$ ) of this makes sense (since a count of aircraft and people cannot practically be fractional), since this is actually a rate, it will not be rounded so that any subsequent calculations are not affected.

Equation (20) also demonstrates one possible measure of an individual aircraft's efficiency.

\section{Performance Metrics}

The primary mathematical evaluation tool developed for quantitative analysis is an extension of development by McGovern and Gupta [14]. As shown in Formula (21) and subsequently referred to as the efficacy index EI, it is the ratio of the difference between a calculated measure $x$ and its worst-case measure $x_{\min }$ to the measure's sample range (i.e., the difference between the best-case measure $x_{\max }$ and the worst-case measure as given by: $\max \left(\mathrm{X}_{\mathrm{y}}\right)-\min \left(\mathrm{X}_{\mathrm{z}}\right): \mathrm{y}, \mathrm{z} \in\{1,2, \ldots,|\mathrm{X}|\}$ from the area of statistical quality control). It is expressed as a percentage and described by

$$
E I_{x}=\frac{100 \cdot\left(x-x_{\min }\right)}{x_{\max }-x_{\min }}
$$

This generates a value between zero and $100 \%$, indicating the percentage of optimum for any given measure and any given combinatorial optimization methodology being evaluated. For example, the efficacy index formula for an airport's aircraft capacity would read

$$
E I_{A C A P}=\frac{100 \cdot\left(A C A P-A C A P_{\text {min }}\right)}{A C A P_{\text {max }}-A C A P_{\text {min }}}
$$

For the study of multiple data sets, probability theory presents us with the concept of a sample mean. The sample mean of a method's efficacy index can be calculated using

$$
\overline{E I}_{x}=\left(\sum_{i=1}^{y} \frac{100 \cdot\left(x_{i}-x_{\min }\right)}{x_{\max }-x_{\min }}\right) / y
$$


where $y$ is the sample size (the number of data sets). While Formula (21) provides individual data set size efficacy indices - especially useful in demonstrating worst and best case as well as trends with instance size - Formula (23) allows a single numerical value that provides a quantitative measure of the location of the data center in a sample.

\section{Case Study}

Any actual study needs to use empirical data that matches a particular airport including fleet mix and aircraft characteristics. Here, a sample case study is provided to demonstrate the application of some of these formulae and to generate some characteristic results. Analysis is conducted using the model and bounds to quantitatively depict the impact of changes in various parameters.

This case study makes use of data from an actual airport - George Bush Intercontinental Airport (KIAH) in Houston, Texas - from a previously conducted study [15]. Items used from this study are shown in Table 1 and include aircraft types, frequencies (based on the fleet mix at that airport), and approach airspeed distributions (collected through the use of multiple pilots flying multiple approaches in Level D simulators). The aircraft types are masked here to avoid the possibility of making any inferences about individual airframes or manufacturers, but include 11 aircraft types (which, in turn, are used to represent 38 different types in the original study, from a total fleet mix of 47 at KIAH) from seven manufacturers. The fictitious capacity improvement in this case study will simply consist of some procedure or technology that is being considered that would have the aircraft fly faster on the entirety of the approach. This procedure/technology would take the aircraft from each of their current approach airspeed modes (i.e., the speed each type flies the most often) to its highest airspeed seen on approach. For example, the current procedures/technologies would have aircraft A fly at 137.42 KIAS, while the new procedure/technology would have that same aircraft fly the approach at 148.60 KIAS (as such, the $V_{\min }$ speeds in Table 1 are not used here and are included only as supplementary information).
Table 1. KIAH Representative Aircraft and Associated Approach Data

\begin{tabular}{|c|c|r|r|l|}
\hline A/C Type & Frequency & \multicolumn{1}{|c|}{$\boldsymbol{V}_{\min }$} & Mode $\boldsymbol{V}$ & $\boldsymbol{V}_{\max }$ \\
\hline $\mathrm{A}$ & 54 & 129.30 & 137.42 & 148.60 \\
\hline $\mathrm{B}$ & 8 & 103.60 & 104.40 & 121.80 \\
\hline $\mathrm{C}$ & 11 & 137.80 & 139.02 & 157.30 \\
\hline $\mathrm{D}$ & 305 & 133.70 & 138.91 & 152.60 \\
\hline $\mathrm{E}$ & 300 & 134.60 & 146.45 & 154.40 \\
\hline $\mathrm{F}$ & 70 & 132.50 & 136.84 & 148.90 \\
\hline $\mathrm{G}$ & 41 & 137.20 & 142.02 & 149.40 \\
\hline $\mathrm{H}$ & 759 & 91.41 & 117.80 & 141.80 \\
\hline $\mathrm{I}$ & 87 & 104.33 & 121.40 & 148.80 \\
\hline $\mathrm{J}$ & 40 & 91.41 & 117.80 & 141.80 \\
\hline $\mathrm{K}$ & 94 & 109.30 & 126.52 & 145.20 \\
\hline
\end{tabular}

Each aircraft's associated length (length in NM) is listed in Table 2. Using this data and the highest speeds as found in Table 1, each possible $A C A P_{\max }$ value can be calculated (see Table 2) using equation (20). $A C A P_{\min }$ is zero from equation (18).

Table 2. Capacity Bounds using KIAH Data

\begin{tabular}{|c|r|r|}
\hline A/C Type & length $\mathbf{( N M )}$ & $\boldsymbol{A C A P}_{\max }$ \\
\hline A & 0.0203 & $7,325.18$ \\
\hline B & 0.0122 & $9,950.31$ \\
\hline C & 0.0252 & $6,238.11$ \\
\hline D & 0.0197 & $7,753.50$ \\
\hline E & 0.0213 & $7,244.71$ \\
\hline F & 0.0256 & $5,827.62$ \\
\hline G & 0.0399 & $3,744.10$ \\
\hline H & 0.0142 & $9,970.14$ \\
\hline I & 0.0192 & $7,756.19$ \\
\hline J & 0.0243 & $5,828.09$ \\
\hline K & 0.0107 & $13,629.52$ \\
\hline
\end{tabular}

Table 2 indicates that aircraft type $\mathrm{K}$ provides the largest $A C A P_{\max }$ for the case study. Even though it is one of the slower aircraft, its small size compensates significantly for its slightly lower speed in this case. For this example, the maximum theoretical aircraft capacity is equal to $13,629.52$ aircraft per hour.

The actual $A C A P$ value (prior to any procedure or technology changes) at KIAH is determined in Table 3 by using $V_{e}$ s equal to the Table 1 mode $V$ values and then weighting each aircraft's calculated $\mu$ (now using the actual $3 \mathrm{NM}$ separation and setting the aircraft lengths to zero) using equation (15). This 
is done by multiplying each aircraft's calculated $\mu$ (used interchangeably with $A C A P$ ) by its frequency (given in Table 1) divided by the total number of aircraft (1769 in this example). Summing these values gives an initial actual $A C A P$ of 42.97 aircraft per hour. Using equation (22), an $E I_{A C A P}$ of less than one percent $(0.32 \%)$ is calculated, indicating the significant spacing between aircraft relative to the aircrafts' sizes.

Table 3. Baseline Service Rate Values for Individual Aircraft

\begin{tabular}{|c|l|r|}
\hline A/C Type & $\boldsymbol{\mu}$ & Weighted $\boldsymbol{\mu}$ \\
\hline A & 45.81 & 1.40 \\
\hline B & 34.80 & 0.16 \\
\hline C & 46.34 & 0.29 \\
\hline D & 46.30 & 7.98 \\
\hline E & 48.82 & 8.28 \\
\hline F & 45.61 & 1.80 \\
\hline G & 47.34 & 1.10 \\
\hline H & 39.27 & 16.85 \\
\hline I & 40.47 & 1.99 \\
\hline J & 39.27 & 0.89 \\
\hline K & 42.17 & 2.24 \\
\hline
\end{tabular}

Assuming the addition of a technology or procedure that safely increases the aircraft approach speeds (all else in this example, including interval between aircraft, remains unchanged), and using the maximum approach speeds to provide representative empirical data, the new $A C A P$ value can be determined. Using the same procedure described for Table 3, the calculations in Table 4 give a modified procedure/technology actual ACAP of 49.00, indicating a capacity increase of an additional six aircraft per hour. However, using equation (22) an $E I_{A C A P}$ of $0.36 \%$ is calculated; just a $0.04 \%$ improvement over the original $E I_{A C A P}$, even though the relative improvement between $A C A P$ values (i.e., from 42.97 to 49.00 ) is over $14 \%$.
Table 4. Modified Service Rate Values for Individual Aircraft

\begin{tabular}{|c|l|r|}
\hline A/C Type & $\boldsymbol{\mu}$ & Weighted $\boldsymbol{\mu}$ \\
\hline $\mathrm{A}$ & 49.53 & 1.51 \\
\hline $\mathrm{B}$ & 40.60 & 0.18 \\
\hline $\mathrm{C}$ & 52.43 & 0.33 \\
\hline $\mathrm{D}$ & 50.87 & 8.77 \\
\hline E & 51.47 & 8.73 \\
\hline F & 49.63 & 1.96 \\
\hline G & 49.80 & 1.15 \\
\hline H & 47.27 & 20.28 \\
\hline I & 49.60 & 2.44 \\
\hline J & 47.27 & 1.07 \\
\hline K & 48.40 & 2.57 \\
\hline
\end{tabular}

Next, the $M / D / 1$ model can be used to compare the system before and after the procedure/technology changes. Using a fixed arrival rate of $\lambda=35$ aircraft per hour and formulae (3) through (8), Table 5 shows that the number of aircraft waiting for approach clearance (i.e., slowing, holding, vectored for spacing, etc.) drops almost in half (per the $L_{q}$ value), while slight decreases in waiting times $\left(W_{q}\right)$ are also seen.

Table 5. Summary of Before and After Results

\begin{tabular}{|l|r|r|}
\hline Parameters & \multicolumn{1}{|c|}{ Baseline } & \multicolumn{1}{c|}{ Modified } \\
\hline$A C A P($ also $\mu)$ & 42.97 & 49.00 \\
\hline$\rho$ & 0.81 & 0.71 \\
\hline$L$ & 2.60 & 1.61 \\
\hline$L_{q}$ & 1.79 & 0.89 \\
\hline$W$ & 0.07 & 0.05 \\
\hline$W_{q}$ & 0.05 & 0.03 \\
\hline$P_{0}$ & $18.56 \%$ & $28.57 \%$ \\
\hline$E I_{A C A P}$ & $0.32 \%$ & $0.36 \%$ \\
\hline
\end{tabular}

This case study hypothetical procedure/ technology adds a full six aircraft per hour, per runway to the airport capacity, while simultaneously decreasing the number of aircraft being delayed for approach clearance as well as the amount of time those aircraft spend waiting; however, the improvement is not as impressive when compared to the upper and lower theoretical bounds. 
Finally, other studies could evaluate various combinations of varying approach speeds, aircraft sizes, fleet mixes, spacing between aircraft, passenger loads, arrival rates, etc. Also, any results using these parameters could then be subsequently validated using simulation.

\section{Summary}

Airport capacity-improving procedures and technologies may need to be evaluated prior to implementing any changes in the national airspace system. In this paper, terminal area upper and lower capacity bounds were developed along with formulations for measuring and for benchmarking capacity improvements for the purpose of quantifying improvements due to the proposed technologies/ procedures prior to their acquisition and implementation. In order to demonstrate these formulations, the terminal area was represented using an $M / D / 1$ model from the field of queueing theory. A case study was then developed, demonstrating a fictional proposed improvement and the numerical results. These mathematical tools may hold value in measuring potential improvements to the NAS as part of a cost-benefit analysis or in other studies.

\section{References}

[1] Federal Aviation Administration, 2009, FAA's NextGen Implementation Plan, Washington, D.C., NextGen Integration and Implementation Office.

[2] Ginsburg, A., 2009, Queuing Theory Applications on Runway Capacity and Priorities, Probabilistic Operations Research IEMG230, April 15, Boston, MA, Northeastern University.

[3] Newell, G. F., 1979, “Airport Capacity and Delays," Transportation Science, 13(3), Hanover, MD, INFORMS, pp. 201-241.

[4] Beasley, J. E., M. Krishnamoorthy, Y. M. Sharaiha, and D. Abramson, 2000, "Scheduling Aircraft Landings - The Static Case," Transportation Science, 34(2), Hanover, MD, INFORMS, pp. 180197.

[5] Janic, M., 2005, "Modeling the Large Scale Disruptions of an Airline Network," Journal of Transportation Engineering, April, Reston, VA, ASCE, pp. 249-260.
[6] Atkin, J.A.D., E. K. Burke, J. S. Greenwood, and D. Reeson, 2007, "Hybrid Metaheuristics to Aid Runway Scheduling at London Heathrow Airport," Transportation Science, 41(1), Hanover, MD, INFORMS, pp. 90-106.

[7] Venkatakrishnan, C. S., A. Barnett, and A. R. Odoni, 1993, "Landings at Logan Airport: Describing and Increasing Airport Capacity," Transportation Science, 27(3), Hanover, MD, INFORMS, pp. 211227.

[8] Hillier, F. S. and Lieberman, G. J., 2004, Introduction to Operations Research, $8^{\text {th }}$ Edition, New York, NY, McGraw-Hill.

[9] Pinedo, M., 2002, Scheduling Theory, Algorithms and Systems, Upper Saddle River, NJ, Prentice-Hall.

[10] Vincenty, T., 1975, "Direct and Inverse Solutions of Geodesics on the Ellipsoid with Application of Nested Equations," Survey Review, XXIII(176), Surrey, UK, Directorate of Overseas Surveys of the Ministry of Overseas Development, pp. 88-93.

[11] McGovern, S. M. and S. G. Creaghan, 2006, "Simple Methodology for the Stochastic Independent Event Calculation of Air Traffic Conflicts,"

Proceedings of the NASA Integrated

Communication, Navigation, and Surveillance

Conference \& Workshop 2006, April 30 - May 3, Baltimore, MD, NASA, CD-ROM.

[12] U.S. Navy, 1984, NATOPS Flight Manual Navy Model T-34C Aircraft, Change 1 1986, Philadelphia, PA, Naval Air Technical Services.

[13] Harrison, M. J., 2006, “Air-to-Air Avionics Integration," Proceedings of the NASA Integrated Communication, Navigation, and Surveillance Conference \& Workshop 2006, April 30 - May 3, Baltimore, MD, NASA, CD-ROM.

[14] McGovern, S. M. and S.M. Gupta, 2007, "Combinatorial Optimization Analysis of the Unary NP-Complete Disassembly Line Balancing Problem," International Journal of Production Research, 45(1819), London, UK, Taylor \& Francis, pp. 4485-4511.

[15] McCartor, G. R. and S. Ladecky, 2005, Safety Study Report on Triple Simultaneous Parallel Instrument Landing System and Area Navigation/ Required Navigation Performance Approaches at 
George Bush Intercontinental Airport, DOT-FAAAFS-440-16, Oklahoma City, OK, FAA.

\section{Acknowledgements}

This material is based on work supported under RITA/VNTSC \#FA7GB1 FD4RN.

\section{Disclaimer}

Any opinions, findings, and conclusions or recommendations expressed in this paper are those of the author.

28th Digital Avionics Systems Conference October 25-29, 2009 